\title{
TUMOR NEUROEPITELIAL DISEMBRIOPLÁSTICO ESTUDO EPIDEMIOLÓGICO DE UMA ÚNICA INSTITUIÇÃO
}

\author{
SÉRGIO ROSEMBERG*, GLACY S. VIEIRA**
}

\begin{abstract}
RESUMO - A real incidência do tumor neuroepitelial disembrioplástico (DNT) é desconhecida. Diferentes estimações foram feitas, de acordo com o tipo de recrutamento dos pacientes. Nas séries provenientes de pacientes operados por epilepsia fármaco-resistente, as taxas variam amplamente, de $5 \%$ a $87 \%$. Em uma série de 600 tumores pediátricos do sistema nervoso central, $9 \mathrm{DNT}$ foram encontrados $(1,5 \%)$. Como o DNT foi identificado apenas em 1988, revimos nossos casos (1975-1991) de gangliogliomas $(n=25)$, oligoastrocitomas $(n=9)$, oligodendrogliomas do lobo temporal $(n=11)$, astrocitomas grau II (OMS 1993) $(n=44)$, independentemente da idade dos pacientes, bem como todos os astrocitomas grau II $(n=61)$ e oligodendrogliomas $(n=10)$ em pacientes até a idade de 20 anos. Sete DNT foram encontrados. Quatro haviam sido previamente diagnosticados como gangliogliomas e 3, como astrocitomas grau II. A partir de 1992, 4 outros DNT foram diagnosticados, perfazendo um total de 11 casos. Oito pacientes tinham menos de 17 anos (6 a 17, média 10,3 anos) e três tinham 27, 42 e 51 anos. Oito tumores eram temporais, 1 frontal e 2 occipitais. Todos os pacientes apresentavam epilepsia fármacoresistente. Dentre todos os tumores neuroepiteliais diagnosticados em nosso Serviço nos últimos 22 anos, DNT compreedem $1,2 \%$ em pacientes até 20 anos $(n=660), 0,24 \%$ em pacientes acima de 20 anos $(n=1254)$, e $0,63 \%$ na totalidade dos pacientes $(\mathrm{n}=1914)$.
\end{abstract}

PALAVRAS-CHAVE: tumor neuroepitelial disembrioplástico, neoplasias do sistema nervoso central, epidemiologia, cirurgia da epilepsia.

\section{Dysembryoplastic neuroepithelial tumor: an epidemiological study from a single institution}

ABSTRACT - The true incidence of dysembryoplastic neuroepithelial tumor (DNT) is unknown. Variable estimations have been advanced according to the type of recruitment of patients. In series of patients with tumors who were operated on for pharmacoresistant epilepsy, the rates varied widely, from 5\% to $87 \%$. Among 600 pediatric tumors in a series, 9 DNT (1.5\%) were found. Since DNT was identified only in 1988, we reviewed our cases $(1975-1991)$ of gangliogliomas $(n=25)$, oligoastrocytomas $(n=9)$, temporal oligodendrogliomas $(n=11)$, temporal astrocytomas grade II (OMS 1993) $(n=44)$ irrespective to age, as well as all astrocytomas grade II $(n=61)$ and oligodendrogliomas $(n=10)$ in patients up to 20 years of age. Seven DNT were encountered. Four cases had been formerly diagnosed as gangliogliomas and 3 as astrocytomas grade II. From 1992 on, 4 more DNT were diagnosed, making up a total of 11 cases. Eight patients were under age 17 (6y to $17 \mathrm{y}$, mean $10.3 \mathrm{y}$ ) and 3 were 27, 42 and 51 year-old. Eight tumors were temporal, 1 frontal and 2 occipital. All patients have had pharmacoresistant epilepsy. Among all neuroepithelial tumors diagnosed in our Division in the last 22 years, DNT comprises $1.2 \%$ in patients under age $20(\mathrm{n}=660), 0.24 \%$ in patients over 20 years $(\mathrm{n}=1254)$, and $0.63 \%$ all ages considered $(\mathrm{n}=1914)$.

KEY WORDS: dysembryoplastic neuroepithelial tumor, central nervous system neoplasms, epidemiology, surgery of epilepsy.

Tumor neuroepitelial disembrioplástico (DNT) é entidade anátomo-clínica de reconhecimento relativamente recente ${ }^{1}$, tendo sido incluída na nova classificação histológica de tumores do sistema nervoso da Organização Mundial de Saúde dentro da categoria "tumores neuronais e neurogliais

Departamento de Patologia, Disciplina de Neuropatologia, Faculdade de Medicina da Universidade de São Paulo (FMUSP): *Professor Associado, *Pós-graduando. Aceite: 13-janeiro-1998.

Dr. Sérgio Rosenberg - Departamento de Patologia. FMUSP - Av. Dr. Arnaldo 455 - 01246-903 São Paulo SP - Brasil. 
mistos"2. Trata-se de lesão indolente, estável, sem efeito de massa ou edema, sempre praticamente restrita ao córtex e de localização preferencial no lobo temporal, ocorrendo sobretudo em indivíduos jovens, abaixo dos 20 anos de idade. Caracteristicamente, apresenta-se, sob o ponto de vista clínico, como crises parciais complexas de longa duração, associadas ou não a crises generalizadas. $\mathrm{O}$ prognóstico é excelente uma vez que sua extirpação leva à cura, sem recidivas ${ }^{3}$. A recente caracterização desses tumores e o aumento progressivo de casos diagnosticados deve-se, sem dúvida, à popularização da utilização da ressonância magnética na epilepsia o que permitiu sua perfeita visualização e à multiplicação dos serviços devotados à neurocirurgia funcional. Em que pese o fato de, nos últimos anos, a literatura relativa a esta entidade ser relativamente abundante, não há estimativas precisas quanto à sua incidência, uma vez que as poucas casuísticas compreendendo séries numerosas são discordantes em seus resultados. Assim, números díspares como $5 \% 4,8 \%$, $23 \%{ }^{6}$ e $87 \%^{7}$ têm sido avançados, devendo esta variação ao tipo de recrutamento dos pacientes. Entre 600 tumores pediátricos do SNC, Taratuto et al. ${ }^{8}$ encontraram 9 DNT, ou seja $1.5 \%$.

O objetivo deste trabalho é o de estudar a incidência de DNT em relação aos tumores neuroectodérmicos do sistema nervoso central diagnosticados na Divisão de Anatomia Patológica do Hospital das Clínicas da Faculdade de Medicina da Universidade de São Paulo (HC/FMUSP).

\section{MATERIAL E MÉTODO}

Como o DNT foi identificado em 1988 e o primeiro caso de nossa série diagnosticado como tal data de 1992, foram revistos todos os casos diagnosticados na Divisão de Anatomia Patológica do HC/FMUSP, entre 1975 e 1991, de tumores que se prestam ao diagnóstico diferencial dos DNT, a saber, gangliogliomas, oligoastrocitomas, oligodendrogliomas dos lobos temporais, astrocitomas (grau II, OMS 1993) dos lobos temporais, independentemente da idade do paciente. Foram também revistos os astrocitomas grau II e oligodendrogliomas de quaisquer localizações, em pacientes abaixo de 20 anos de idade. O critério histológico para o diagnóstico de

Tabela 1. Dados clínicos e diagnóstico prévio em 11 casos de DNT.

\begin{tabular}{|c|c|c|c|c|c|}
\hline Caso & $\begin{array}{l}\text { Idade } \\
\text { (anos) }\end{array}$ & Sexo & Localização & $\begin{array}{c}\text { Diagnóstico } \\
\text { Prévio }\end{array}$ & $\begin{array}{l}\text { Quadro } \\
\text { Clínico }\end{array}$ \\
\hline 1 & 9 & $\mathrm{~F}$ & Frontal D & Astrocitoma & CPC desde os 5 anos \\
\hline 2 & 16 & M & Temporal E & Astrocitoma & Epilepsia focal desde 18 meses \\
\hline 3 & 13 & $\mathrm{~F}$ & Temporal D & Ganglioglioma & Crises focais desde a infância \\
\hline 4 & 11 & M & Occipital E & Ganglioglioma & Crises focais desde os 4 anos \\
\hline 5 & 9 & M & Temporal D & Ganglioglioma & CPC desde os 2 anos \\
\hline 6 & 17 & M & Temporal E & Astrocitoma & CPC desde os 12 anos \\
\hline 7 & 43 & M & Temporal E & Ganglioglioma & $\mathrm{CPC}$ há 8 meses antes da cirurgia \\
\hline 8 & 51 & M & Temporal D & DNT & CPC desde os 42 anos \\
\hline 9 & 11 & M & Occipital E & DNT & Cirses focais desde os 3 anos \\
\hline 10 & 7 & M & Temporal D & DNT & Crises focais desde os 3 anos \\
\hline 11 & 27 & M & Temporal E & DNT & CPC desde os 11 anos \\
\hline
\end{tabular}




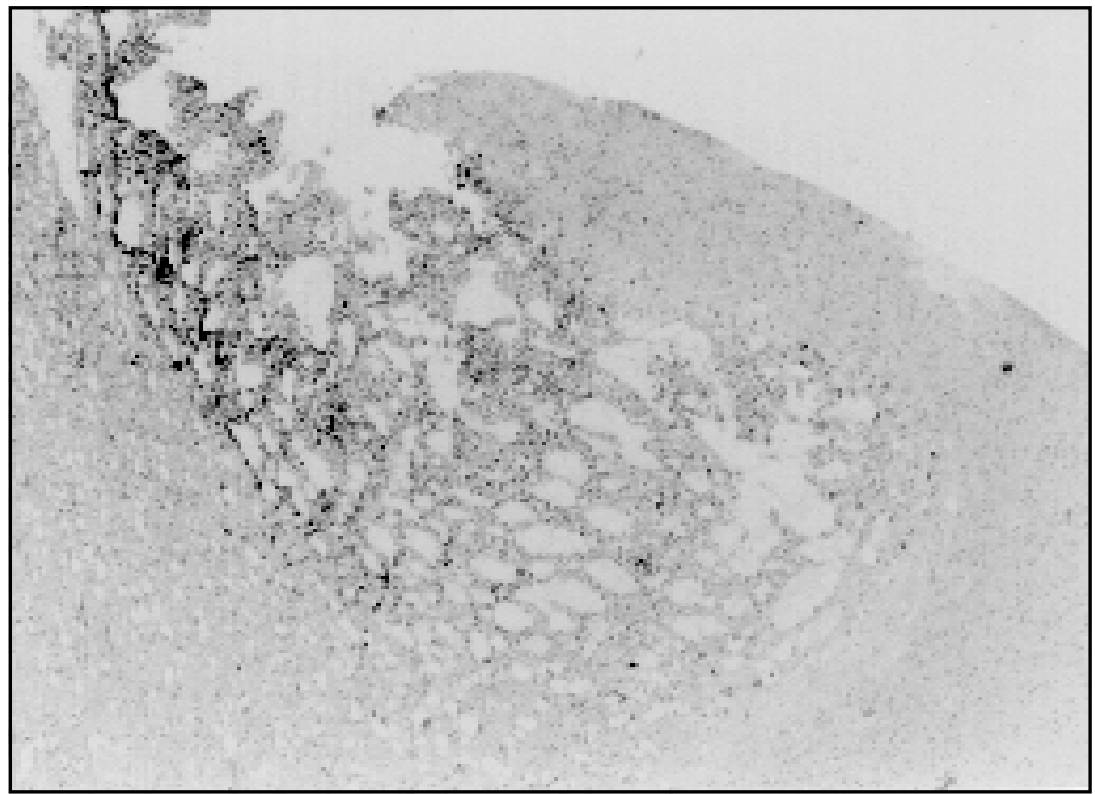

Fig 1. DNT. Arranjo nodular da lesão intra-cortical. HE X60.

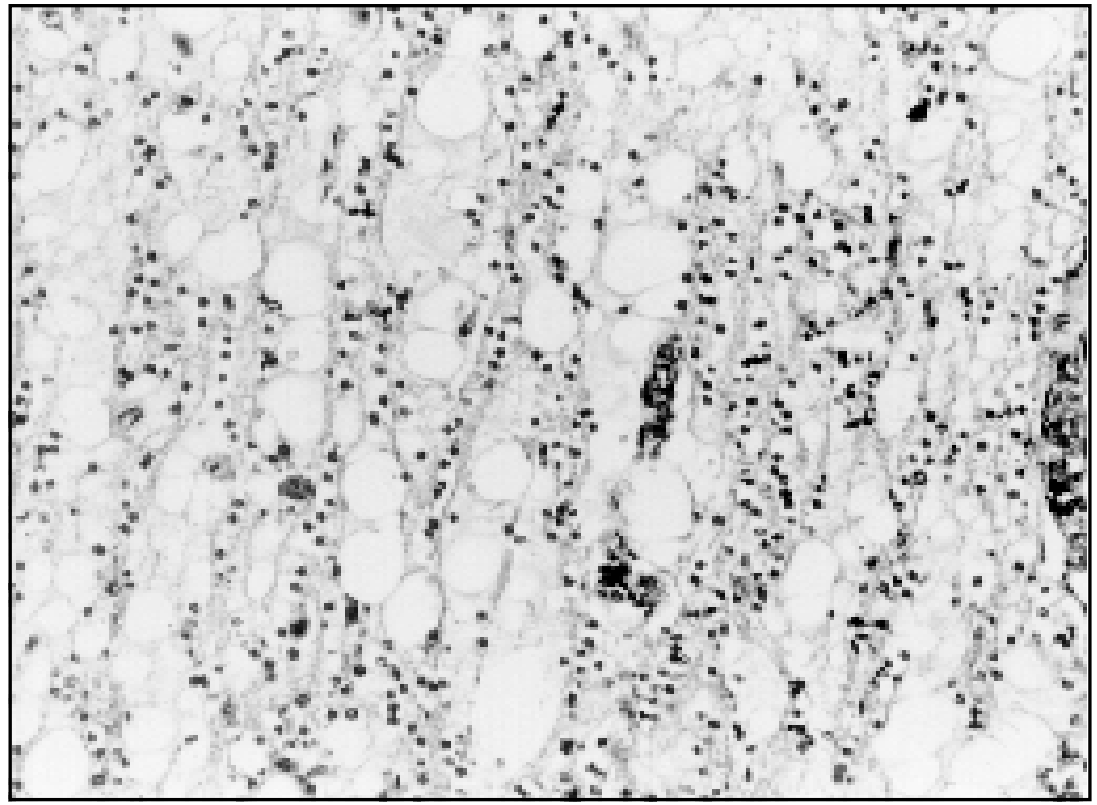

Fig 2. DNT. Elemento glioneural específico. Observar o arranjo colunar característico. HE X 250 
DNT foi bastante restrito, exigindo-se sempre o encontro do elemento glio-neural específico, ou seja a presença de células oligodendrogliais-símiles dispostas em cordões ao longo de estruturas axonais . Em todos os casos, células neuro-ganglionares que "flutuavam" num lago de mucina, estavam presentes (Fig 1). Em praticamente todos os casos, um arranjo nodular característico foi encontrado (Fig 2). O reconhecimento da existência, bastante frequente nestes tumores, de displasia do córtex adjacente à lesão, o qual depende da quantidade do material enviado ao exame histológico e à sua orientação apropriada, não foi considerado critério necessário para o diagnóstico, estando presente numa minoria de casos.

\section{RESULTADOS}

Cento e sessenta neoplasias foram revistas, assim distribuídas: 25 gangliogliomas, 9 oligoastrocitomas, 11 oligodendrogliomas temporais, 44 astrocitomas grau II dos lobos temporais em paciente de qualquer idade, 61 astrocitomas grau II e 10 oligodendrogliomas de qualquer localização em pacientes abaixo de 20 anos de idade. Dentre estes tumores foram encontrados 7 DNT, sendo que o diagnóstico prévio havia sido ganglioglioma em 4 e astrocitoma grau II, em 3 casos. A partir de 1992, mais 4 DNT foram diagnosticados, perfazendo um total de 11 casos. Os dados clínicos relativos a esses pacientes estão listados na Tabela 1. Oito pacientes tinham menos de $17 \operatorname{anos}(6$ a 17, média 10,3 anos) e três tinham 27, 42 e 51 anos de idade, respectivamente. Oito tumores situavam-se nos lobos temporais, um no lobo frontal e dois, no occipital. Todos os pacientes tinham epilepsia fármaco-resistente de longa duração (média: 7,9 anos).

Entre todos os tumores neuroepiteliais diagnosticados neste Serviço entre 1975 e 1996 em indivíduos abaixo de 20 anos (660 casos), os DNT correspondem a 1,2\%. Nos pacientes com mais de 20 anos (1254 casos), os DNT representam 0,24\%. Este número sobe a 0,63\%, todas as idades consideradas (1914 casos).

\section{DISCUSSÃO}

Se bem que mais de 300 casos de DNT tenham sido relatados na literatura ${ }^{9}$, a verdadeira incidência desta neoplasia permanece ignorada, devido principalmente a dois fatores. Em primeiro lugar, o tipo de recrutamento dos pacientes é determinante: os DNT tendem a ser mais numerosos naquelas séries cujos pacientes são provenientes de serviços devotados à cirurgia da epilepsia. Mesmo assim, as cifras podem variar de 5 a $23 \%$, atingindo na série de Kirkpatrick et al ${ }^{7}$. o número surpreendente de $87 \%$. Esta disparidade se deve, sem dúvida, ao segundo problema que é a caracterização histológica dos DNT. Desde a sua descrição em 1988, os critérios histológicos diagnósticos têm evoluído, sendo que atualmente a maior parte dos neuropatologistas esteja de acordo para considerar a existência de pelo menos duas formas de DNT, a saber, uma forma "simples" composta unicamente pelo elemento glioneuronal específico (o mais apto a ser confundido com outras neoplasias gliais, notadamente astrocitomas e oligodendrogliomas), e uma forma "complexa" caracterizada pelo arranjo nodular do elemento glioneuronal específico associado à presença de áreas francamente astrocitárias e/ou oligodendrogliais. Uma terceira forma dita "não específica" foi recentemente proposta ${ }^{10}$, na qual não existe o elemento glioneuronal específico, sendo o tumor caracterizado por critérios radiológicos (lesão cortical, sem efeito de massa e sem progressão) cuja histologia pode ser idêntica a astrocitomas pilocíticos, oligodendrogliomas ou oligoastrocitomas. Em nosso método, exigiu-se a presença do elemento glioneuronal específico conforme exposto, sendo que, na quase totalidade dos casos, o arranjo nodular estava presente.

Na única série na literatura comparável à nossa, em que um estudo retrospectivo de 600 casos de tumores neuroepiteliais pediátricos foram analisados, os resultados foram bastante semelhantes $\left(1,5 \%\right.$ na série de Taratuto et al. $\left.{ }^{8}\right)$

O fato de mais de um terço dos casos de nossa série terem sido diagnosticados nos últimos cinco anos é devido, sem dúvida, ao uso praticamente sistemático da ressonância magnética em pacientes com epilepsia fármaco-resistente e ao incremento em nossa Instituição da cirurgia da epilepsia. 
Finalmente, deve-se considerar ser possível que a real frequência de DNT seja ligeiramente superior àquela por nós encontrada, se não tivéssemos limitado a revisão por faixa etária ou por topografia, como fizemos com alguns tipos de tumores.

\section{REFERÊNCIAS}

1. Daumas-Duport C, Scheithauer BW, Chodkiewicz JP, Laws ER, Vedrenne C. Dysembryoplastic neuroepithelial tumor: a surgically curable tumor of young patients with intractable partial seizures. Report of thirty-nine cases. Neurosurgery 1988;23:545-556.

2. Kleihues P, Burger PC, Scheithauer BW. Histological typing of tumours of the central nervous system. 2 Ed., Berlin: Springer-Verlag, 1993.

3. Daumas-Duport C. Dysembryoplastic neuroepithelial tumor. Brain Pathol 1993;3:283-295.

4. Morris HH, Estes ML, Gilmore R, Van-Ness PC, Barnett GH, Turnbull J. Chronic intractable epilepsy as the only symptom of primary brain tumor. Epilepsia 1993;34:1038-1043.

5. Wolf Hk. Campos MG, Zentner J, Hufnagel A, Schramm J, Elger CE, Wiestler OD. Surgical pathology of temporal lobe epilepsy. J Neuropathol Exp Neurol 1993;52:499-506.

6. Prayson RA, Estes ML. Cortical dysplasia: a histopathologic study of 52 cases of partial lobectomy in patients with epilepsy. Hum Pathol 1995; 26:493-500.

7. Kirkpatrick PJ, Honavar M, Janota I, Polkey CE. Control of temporal lobe epilepsy following en bloc resection of lowgrade tumors. J Neurosurg 1993;78:19-25.

8. Taratuto AL,Pomata H, Sevlever G, Gallo G, Monges J. Dysembryoplastic neuroepithelial tumor: morphological, immunocytochemical and deoxyribonucleic acid analysis in a pediatric series. Neurosurgery 1995;36:474-481.

9. Kleihues P, Cavenee WK. Pathology and genetics of tumours of the nervous system. Lyon:International Agency for Research on Cancer, 1997.

10. Daumas-Duport C. Dysembryoplastic neuroepithelial tumours in epilepsy surgery, in Guerrini R (ed): Dysplasia of Cerebral Cortex and Epilepsy. New-York: Raven Press, 1995,pp125-147. 